\title{
BMJ Open Cancer-related information needs and treatment decision-making experiences of people with dementia in England: a multiple perspective qualitative study
}

\author{
Lorna McWilliams, ${ }^{1,2}$ Carole Farrell, ${ }^{1,2}$ John Keady, ${ }^{2}$ Caroline Swarbrick, ${ }^{2}$ \\ Lorraine Burgess, ${ }^{3}$ Gunn Grande, ${ }^{2}$ Sarah Bellhouse, ${ }^{1}$ Janelle Yorke ${ }^{1,2}$
}

To cite: McWilliams L, Farrell C, Keady J, et al. Cancer-related information needs and treatment decision-making experiences of people with dementia in England: a multiple perspective qualitative study. BMJ Open 2018;8:e020250. doi:10.1136/ bmjopen-2017-020250

\section{- Prepublication history and} additional material for this paper are available online. To view these files, please visit the journal online (http://dx.doi. org/10.1136/bmjopen-2017020250).

Received 25 October 2017 Revised 30 January 2018 Accepted 1 February 2018
Check for updates

${ }^{1}$ Christie Patient Centred Research, School of Oncology, The Christie NHS Foundation Trust, Manchester, UK

${ }^{2}$ Division of Nursing, Midwifery and Social Work, School of Health Sciences, University of Manchester, Manchester, UK ${ }^{3}$ Psycho-Oncology, The Christie NHS Foundation Trust, Manchester, UK

\section{Correspondence to}

Dr Lorna McWilliams;

lorna.mcwilliams@christie.

nhs.uk

\section{ABSTRACT}

Objectives Little is known about the cancer experience and support needs of people with dementia. In particular, no evidence currently exists to demonstrate the likely complex decision-making processes for this patient group and the oncology healthcare professionals (HCP) involved in their care. The aim of this study was to explore the cancer-related information needs and decision-making experiences of patients with cancer and comorbid dementia, their informal caregivers and oncology HCPs.

Design Cross-sectional qualitative study. Semistructured interviews were conducted face to face with participants. Interviews were audio recorded and transcribed prior to thematic analysis

Setting Patients with a diagnosis of cancer and dementia their informal caregivers and oncology HCPs involved in their care, all recruited from a regional treatment cancer centre.

Participants Purposeful sample of 10 patients with a diagnosis of cancer-dementia, informal caregivers $(n=9)$ and oncology HCPs $(n=12)$.

Results Four themes were identified: (1) leading to the initial consultation-HCPs require more detailed information on the functional impact of dementia and how it may influence cancer treatment options prior to meeting the patient; (2) communicating clinically relevant information-informal caregivers are relied on to provide patient information, advocate for the patient and support decision-making; (3) adjustments to cancer care-patients with dementia get through treatment with the help of their family and (4) following completion of cancer treatmentthere are continuing information needs. Oncology HCPs discussed their need to consult specialists in dementia care to support treatment decision-making.

Conclusions Although patients with cancer-dementia are involved in their treatment decision-making, informal caregivers are generally crucial in supporting this process. Individual patient needs and circumstances related to their cancer must be considered in the context of dementia prognosis highlighting complexities of decision-making in this population. Oncology teams should strive to involve healthcare staff with dementia expertise as early as possible in the cancer pathway.

\section{Strengths and limitations of this study}

- This is the first report of a multiperspective analysis between patient, informal caregiver and oncology healthcare professionals on the experiences of cancer-dementia and treatment decision-making.

- The sample includes a rich variety of patients who have a diagnosis of cancer-dementia and have undergone cancer treatment decision-making, most often with support from family members.

- The patient sample was recruited from a single cancer treatment hospital; therefore, it is not clear whether treatment decisions are sometimes made prior to referral to tertiary care.

- As our study was cross-sectional, a longer, prospective study would offer further insights.

\section{BACKGROUND}

While there are many positives about people living longer in everyday society, an ageing population brings a number of individual risks and challenges. For example, the Newcastle 85+ study revealed that older people live with at least two chronic conditions which impinge on quality of life and activities of daily living. ${ }^{1}$ It is well established that advancing age remains the highest dementia risk factor, ${ }^{2}$ a position parallel to cancer. $^{34}$ While prevalence and incidence rates have been well studied, limited evidence documents the situation when diagnoses of dementia and cancer coexist and subsequent implications for healthcare services. ${ }^{5}$ However, from the scarce literature, patients with a coexisting diagnosis of cancer and dementia (henceforth referred to as cancerdementia) have greater healthcare needs and poorer clinical outcomes compared with patients with cancer alone. ${ }^{6-8}$

Shared decision-making in the context of cancer can impact on patient quality-of-care perceptions and satisfaction with medical 
consultation communication. ${ }^{9-12}$ However, in the context of cancer-dementia, decisions about cancer treatment are more likely to be complex. Decision-making by people with dementia regarding their healthcare may be compromised due to different levels of understanding about the risks and benefits of treatment options. ${ }^{13} 14$ Family caregivers frequently advocate for their relatives in such situations ${ }^{15}$ and it is known that family members of patients with cancer are generally involved in supporting treatment decision-making. ${ }^{16}$ Similarly, family caregivers of people with dementia play a key role in important decisions, for example, residential care placement ${ }^{17-19}$ and end of life. ${ }^{20}$ Yet, little is known about treatment experiences and decision-making processes of patients, informal caregivers and oncology healthcare professionals (HCP) in the context of cancer-dementia.

Our recent systematic review of cancer-dementia literature found no papers which explored treatment decision-making experiences from the patient or informal caregiver perspective. ${ }^{21}$ An observational study of outpatient oncology consultations between patients with cancer with memory problems, their family members and HCPs reported that family members provided support while patients tended to minimise their memory problems. ${ }^{22}$ However, memory problems are just one facet of dementia and only three patients in the sample had a clinical dementia diagnosis. ${ }^{22}$ Perspectives from oncology HCPs are also important in order to identify clinical issues relating to decision-making within this context. Studies that have attempted to explore HCP cancer-dementia treatment decision-making have been limited to hypothetical emergency care scenarios or specific to suspected breast cancer referrals only. ${ }^{23} 24$

To our knowledge, no published study has explored the experience and needs that a person with dementia may have around cancer treatment decisions and the role that informal caregivers and HCPs have. The aim of the present study was to investigate cancer-related information needs and treatment decision-making experiences of people with cancer-dementia, their informal caregivers and oncology HCPs involved in their care.

\section{METHODS \\ Design}

A cross-sectional qualitative design using semistructured interviews was used to explore cancer decision-making experiences of (1) people with cancer-dementia; (2) informal caregivers and (3) oncology HCPs involved in their care.

\section{Participants and setting}

This study was set in a regional North West England cancer centre serving a population of 3.2 million. Purposeful sampling was used to identify participants with confirmed dementia diagnoses known to the Macmillan dementia consultant nurse (LB) based at the hospital. Inclusion criteria were patients with: (1) any cancer type, (2) any stage of cancer treatment and (3) any dementia type and severity. An appropriate protocol was followed closely by the researcher and nurse to determine capacity to consent to research. ${ }^{25}$ Including people who may not have capacity to consent provides the opportunity to discuss their experiences in a safe environment. The voice of people with dementia is under-represented in research yet exploration of their experiences is essential to develop appropriate support. ${ }^{26}$ Informal caregivers were eligible to participate following patient consent; however, patients were able to participate irrespective of whether an informal caregiver participated. With permission from patients, oncology HCPs from their clinical team were invited to participate, determined where possible by their involvement in treatment decision-making. Consent to participate followed the study protocol for involving adults with and without capacity.

\section{Procedure}

Potential participants were approached by the nurse consultant who provided study information and requested permission for a researcher (LM) to initiate contact and answer any questions. A capacity to consent assessment was conducted following the process advocated by the British Psychological Society. ${ }^{27} 28$ If it was deemed that a patient lacked capacity to consent, personal (eg, family member) or clinical (eg, general practitioner) 'consultees' were given study information and provided written consent to approach the patient.

An interview topic guide was developed focussing on questions about experience of cancer-dementia including cancer symptoms, diagnosis, treatment decision-making and information needs. This was individualised for each patient to allow for meaningful communication with the researcher. Prompts were used to guide the conversation to gauge memory and understanding. Where possible, patients and informal caregivers were asked to think about their involvement in treatment decision-making. A similar guide was developed for HCP interviews. This focused on general experience of patients with cancerdementia before focusing on the patient participant for each case. Once consent was obtained, demographic and clinical information was collected from medical notes (eg, age, cancer information). Interviews were conducted face to face in a quiet hospital room or in the patient's home. With consent, interviews were audio recorded. Patient participants were given the choice of completing their interview alone or with an informal caregiver. Informal caregivers could support the patient interview and participate in a separate interview. Individual interviews with HCPs were undertaken face to face in a hospital office following completion of patient/caregiver data collection. All but one (CF) interview was conducted by LM, both female full-time postdoctoral researchers with experience of qualitative methods.

\section{Data analysis}

Interviews were audio recorded, anonymised and transcribed verbatim; field notes were taken for each 
Table 1 Sample characteristics $(n=31)$

\begin{tabular}{|c|c|c|c|}
\hline Sample characteristic & $\begin{array}{l}\text { Patient with cancer- } \\
\text { dementia }(n=10)\end{array}$ & Informal caregiver $(n=9)$ & Oncology HCP $(n=12)$ \\
\hline Mean age $\pm S D$ (range) years & $73.6 \pm 15.4(39-93)$ & $63.8 \pm 12.3(47-77)^{*}$ & $41.8 \pm 8.3(30-53) \dagger$ \\
\hline $\begin{array}{l}\text { Relationship to patient or } \\
\text { oncology profession }\end{array}$ & $\mathrm{N} / \mathrm{A}$ & $\begin{array}{l}\text { Spouse }(n=5) \\
\text { Son/daughter }(n=4)\end{array}$ & $\begin{array}{l}\text { Oncology allied health professional: } \\
\text { Clinical nurse specialist }(n=2) \\
\text { Specialist radiographer }(n=1) \\
\text { Oncology doctor }(D r) \text { : } \\
\text { Consultant }(n=3) \\
\text { Surgeon }(n=4) \\
\text { Registrar }(n=1) \\
\text { Clinical fellow }(n=1)\end{array}$ \\
\hline
\end{tabular}

*One family member age missing.

†One clinician age missing.

$\mathrm{HCP}$, healthcare professional.

participant (two patients refused to be audio recorded and field notes were taken which were transcribed for inclusion in analysis). The data were analysed using Braun and Clarke's thematic analysis using the principles of the framework approach to organise the initial coding of the data. ${ }^{2930}$ Each transcript was read and reread to get an overall sense of the data. An agreed (LM, SB, JY) set of codes were applied to interviews and entered into the framework matrix for each of the three participant groups. Ongoing analysis and interpretation were discussed at research management meetings; alternative explanations for emergent findings were suggested and discussed. The analysis reported focused on information needs and decision-making in the context of cancer-dementia.

\section{FINDINGS}

\section{Sample}

The sample comprised people with cancer-dementia $(\mathrm{n}=10)$; informal caregivers $(\mathrm{n}=9)$ and oncology HCPs $(\mathrm{n}=12)$ (table 1). All but two patients had a participating family member (plus two family members jointly participated for one patient). Two patients had capacity to give informed consent; proxy consent was provided by a family member (personal consultee) for eight patients who were deemed to lack capacity. One additional patient with cancer-dementia was invited to participate; however, the personal consultee did not consent for the researcher to approach the patient because of communication difficulties. Of the eight audio-recorded patient interviews, five took place as a dyad or triad and ranged from 46 to $62 \mathrm{~min}$; patient alone interviews $(\mathrm{n}=3)$ lasted $10-35 \mathrm{~min}$. All interviews took place face to face. Interviews were conducted at the patient participants' home in seven cases while two patients had interviews in their private hospital room. The remaining patient interview took place in a private room at the hospital during an outpatient appointment. Separate, informal caregiver interviews (two at home, two in a private room in the hospital) lasted 26-49 min and HCP interviews $15-62 \mathrm{~min}$ in a private room in the hospital.
Cancer disease groups included head and neck, colorectal, urology, skin, gynaecology and haematology. Nine patients had a dementia diagnosis prior to attending the cancer centre and one was diagnosed during cancer treatment. Dementia diagnoses included Alzheimer's disease, Pick's disease, vascular and AIDS-related dementia. Patients underwent a range of cancer treatments including curative radiotherapy $(n=4)$, surgery $(n=3)$, palliative chemotherapy $(n=1)$, stem cell transplant $(\mathrm{n}=1)$ and one patient did not have cancer treatment. This level of participant detail was chosen to protect the anonymity of this small patient sample. Interviews took place post-treatment for eight patients; one patient participant was undergoing radiotherapy treatment and one patient did not undergo cancer treatment.

Data are presented as four overarching themes (headings) with 10 subthemes (subheadings); see table 2. The coding used to represent participants is: $\mathrm{P}=$ patient, $\mathrm{C}=$ informal caregiver, $\mathrm{H}=\mathrm{HCP}$; individual study code; $\mathrm{M}=$ male, $\mathrm{F}=$ female and $\mathrm{HCP}$ role or for caregivers, their

Table 2 Thematic framework of findings

Theme Subtheme

(1) Leading up to the initial cancer consultation

(1.1) Transition to tertiary care (1.2) Preparation for the first oncology consultation

(2) Communicating clinically relevant information

(2.1) Taking more time

(2.2) Navigating treatment decisionmaking information

(2.3) Dementia-specific cancer treatment considerations

(2.4) Decision-making agency

$\begin{array}{ll}\text { (3) Adjustments to } & \text { (3.1) Continuity of care } \\ \text { cancer care } & \text { (3.2) Proxy healthcare professionals }\end{array}$

(4) After cancer treatment finishes 
relationship to the patient (eg, 'C003M:son' is the son of participant 003). For confidentiality, names appearing in quotes are pseudonyms.

The four themes are presented as a cancer pathway trajectory: (1) leading up to the initial cancer consultation, (2) communicating clinically relevant information, (3) adjustments to cancer care and (4) after cancer treatment finishes. Informal caregivers played a pivotal role in supporting patients with cancer-dementia. This appeared across all themes reflected in the presentation of findings where the person with cancer-dementia, at times, found it difficult to communicate about their experience.

\section{Theme 1: leading up to the initial cancer consultation}

1.1: Transition to tertiary care

Family members highlighted issues before the initial oncology consultation, a time period that appeared challenging with several accounts of lengthy processes from symptom recognition and further investigations. At times, this was exacerbated due to limited HCP awareness of the potential impact of dementia on cancer diagnostic investigations:

...asking her very complicated instructions which she just wouldn't be able to interpret and do for them...they weren't adapting the care and the responses (C002M:husband)

Here, the role of caregivers in providing support was emphasised in ensuring timely access to cancer specialists 'if I hadn't been there it certainly would have been weeks' (C003M:son). Delayed access made some feel that HCP decisions were made prior to the patient's first cancer consultation '...appointment took at least threeweeks and by the time it had taken I realised, well, they weren't going to do too much'(C010M:son). HCP perceptions about dementia may impact on cancer referral decisions:

The surgeon also said [before referral to tertiary treatment centre]...'we've got you down as somebody with dementia, but you are obviously not very advanced in dementia'...'Well, I was going to say there wasn't any treatment, but maybe I'm going to refer you...for some possible chemotherapy' (C009F:wife)

However, it is worthy to note that it is not easy to collect appropriate information about the patient to guide HCP decision-making prior to the first oncology consultation.

\section{2: Preparation for the first oncology consultation}

Collating healthcare information about the patient's dementia prior to the initial appointment with the patient was valuable to HCPs for multiple reasons: (1) to appropriately organise cancer treatment decision-making processes regarding capacity to consent '... helpful in the sense that I will be prepared to have like a best of interest meeting' (H001F:Dr,surgeon); (2) to feel more prepared in advance about appropriate communication approaches '...could tailor the consultation a bit better maybe and make it more streamlined and a bit smoother for the family as well' (H002F:Dr,registrar); (3) to have insight into the cognitive abilities of the person living with dementia '...it would be nice to know exactly how well they were functioning and what they were able to do before we embark on any treatment...' (H006F:Dr,clinical fellow) and (4) to involve dementia-specific support at the beginning of the pathway: 'If we knew at the time of referral that there were some issues, I think to have the dementia team or the nurse at that [first] appointment would be quite good' (H005M:Dr,surgeon).

Most HCPs relied on an informal caregiver (usually family members) accompanying the patient at the first oncology clinic appointment to receive this information rather than having it for the initial multidisciplinary team (MDT) meeting 'only really what the son told us in terms of what she was able to do, how she was functioning' (H003F:Dr,consultant). Difficulties arose when there was no caregiver who sufficiently knew the patient with dementia:

Sometimes those patients will come with say someone from the care home, and it's usually I find the most junior person that they'll send who has no idea what's wrong with the patient... why have you come with this person? (H005M:Dr,surgeon)

\section{Theme 2: communicating clinically relevant information}

\section{1: Taking more time}

Patients attended the cancer centre for an initial consultation to discuss treatment options. However, the time required for facilitation of communication, information exchange and understanding about treatment options was a point of contention. Although some HCPs acknowledged the extra time required for communicating with this patient group 'it may take longer to get the full information... you need to identify what they know, what they don't know, how much they understand' (H004F:radiographer), caregivers conveyed a different experience:

It's really fast for me....all I could think was well if I wasn't there and you [patient] wouldn't have remembered...for somebody with dementia it needs to be slowed down (C006F:daughter)

This idea of 'slowing down' related to the need to be mindful of information retention 'he needed to have it repeated a few times' (C009F:wife) impacting on patient understanding 'I can listen alright like what you're saying to me now I know that, but in a few minutes I can't tell you what you've said.' (P006M) and what happened when they left the consultation 'she was involved but she forgot when she [came] out and come home' (C001M:husband). In these instances, it was clear that informal caregivers are relied on to support patients with cancer-dementia and to facilitate the consideration of cancer treatment options 'they can sort of plant the seed almost for you while they're at home' (H004F:radiographer). Patients also found this reassuring: 
I can forget a few things and not ask the correct questions...it's good for somebody here to go with me (P005M)

\section{2: Navigating treatment decision-making information}

In some cases, the patient's family was not clear about the proposed treatment from the initial consultation: ' $I$ weren't sure what the treatment was actually going to be, whether it would be radiation, chemo[therapy], or what' (C003M:son). Yet, considerable adjustments had to be made to support treatment compliance: 'I had to come again out of work to go into hospital to take her down to the radiotherapy to make sure and get her through the treatment' (C003M:son). Other times, information about treatment side effects was not adequately explained leading to patients and families misunderstanding the risks:

They didn't say it like it were...we thought there would be one or two [side effects], but I didn't think they'd be as severe as they were (C004M:husband)

Although HCPs provided written information about cancer treatments, this was not always appropriate 'they [had] given us these leaflets; they had to be read for me...'cause I just can't do it' (P006M) which was acknowledged by staff 'I think the [surgery] booklet was slightly confusing because it's a general booklet for all, so it's not a dementia orientated booklet' (H001F:Dr,surgeon). This left family members to explain information provided from clinic appointments 'that's the opportunity for the family hopefully to read over it a few times with them' (H004F:Dr,consultant).

\section{3: Dementia-specific cancer treatment considerations}

The potential impact that dementia has on cancer treatment decision-making was considered in all cases. On some occasions, family members felt the person's dementia directly influenced treatment options:

I think they were thinking because she's got dementia, the 25 [radiotherapy fractions] might have been too much for her (C004F:daughter)

For others, the impact that treatment may have on dementia at the time of decision-making was less clear 'we didn't know whether the treatment would exacerbate Brenda's dementia' (C002M:husband) and presented difficult clinical challenges for oncologists: 'It wouldn't be fair to deny her radical treatment based on the fact that she would find it difficult [due to dementia] but ultimately we were aiming to cure her of her cancer' (H002F:Dr,registrar).

The complexity of cancer treatment decision-making in the context of dementia emphasised the importance of MDTs when considering treatment options although these take place prior to meeting the patient 'we do sit down and we do make decisions and it goes to the wider MDT as well, so it's not just one person making that decision' (H008F:CNS). Clinicians however also needed to incorporate the views of the patient themselves and family members' insight of how certain aspects of dementia, such as behavioural symptoms, may impact on treatment options the pre-warning [about behaviour] from the daughter was helpful for us to get planning properly' (H007M:Dr,surgeon).

\section{4: Decision-making agency}

At times, it was clear that patients were not actively involved in the decision-making process: 'Yeah, they didn't give you any option.' (P006M) and had unanswered questions about treatment information 'Do they take you in for chemo[therapy] or? Even if it's only one dose or? Is it an injections or summat?' (P001F). This echoed HCP reflections of difficulty engaging with patients during decision-making '...knowing whether he had agreed to and had been part of that decision was a difficult thing for me to assess, because I think he was not communicating at all' (H005M:Dr,surgeon). HCPs explained ways in which they tried to involve the patient in decision-making processes 'I [did] try to actually talk to the patient and look at her and talk to her and the husband would answer' (H002M:Dr,consultant) but family members were often the patient's voice. However, they also acknowledged the importance of considering how treatment may impact on informal caregivers:

we have to do the best for the patient because ultimately it's the patient we're treating, but we've still got to be mindful that things fit for the carer (H008F:CNS)

That [treatment decision] might take a little bit longer again because you've got to consider everybody else's opinions in the treatment of that patient (H004F:radiographer)

\section{Theme 3: adjustments to cancer care}

\section{1: Continuity of care}

Adjustments during cancer treatment were often specifically related to the person's dementia. This included changes to language used to describe treatment processes 'explained it [radiotherapy mask material] was like a wet flannel' (C003M:son) and the importance of staff awareness of patients' potential memory issues to support inpatient spells "Yes your family knows you are here and yes, Alistair, Maria [patient's wife] is coming."(C009F:wife). One person with Alzheimer's disease receiving radiotherapy treatment knew when a HCP was aware of his dementia diagnosis because 'they talk to you a bit more slowly' (P006M), which helped him feel more at ease. Changes were sometimes made to 'usual practice' for radiotherapy treatment, for example: 'her doctor realised that to get Brenda through the treatment... it would have to be as an inpatient' (C002M:husband) and preparations for surgical procedures:

...the day case unit who are made aware that this patient needs to be shorter duration on the ward and in theatre (H007M:Dr,surgeon)

Familiarity of staff treating the person with cancerdementia was frequently mentioned as a way to streamline the care processes during treatment ' $i t$ 's a lot of negotiation... sometimes... it's a case of having a familiar face' (H008F:CNS) although this was not something that is easily achieved ' $I$ think with the best will in the world you can't say, well this is 
your doctor you'll see every time you come for an appointment' (H006F:Dr,clinical fellow). The importance of training staff about dementia was therefore emphasised: 'useful to train some people to have more experience treating people with dementia because sometimes it can be stressful... we have to plan every patient properly and give a bit more time' (H007M:Dr,surgeon). When appropriate adjustments were not made for the person with cancer-dementia, some HCPs felt that they could have intervened at an earlier stage to prevent issues arising:

...I wasn't aware of him [patient] and my colleague wasn't, until it came to that crisis management... would've been good to have been involved in the process...we could just keep a closer eye on him (H009F:CNS)

\section{2: Proxy healthcare professionals}

It was clear that family members assumed the role of proxy healthcare professionals, although not necessarily formalised. However, HCPs relied on informal caregivers to support people with cancer-dementia to identify and manage treatment side effects:

I tend to educate the relatives on signs to look out for, what they need to do..."We need you to be active in looking for signs and symptoms of sepsis etc." (H008F:CNS)

Family members undertook this role by supporting practical aspects of cancer treatment 'one of the nurse assistants up here that gave me some useful tips on care' (C002M:husband) and ensured medication adherence 'I had given him what drugs I was told to give him' (C009F:wife). HCPs considered family members to be trustworthy to carry out such tasks:

her husband was so caring, I had no concerns about the safety of her getting the tablets, cause I knew he could give them to her (H004F:Dr,consultant)

\section{Theme 4: after cancer treatment finishes}

\section{1: Ongoing decision-making processes}

People with cancer-dementia made significant life adjustments following cancer treatment including two participants moving into a nursing home after being an inpatient. This time appeared stressful for patients and their family wanting to 'move on' from the cancer centre 'I want to be nearer home' (P002F); in dealing with uncertainty '... the problem now is you feel like you're no further on.... whether it's [cancer] gone' (C004F:daughter) or dilemmas regarding further investigations:

...they want me to go for it but do I want to go for it? Surely that's my choice? I know we're only really going to know if I go for the biopsy, the scan, but do I really want to know that? (P005M)

\section{2: Lasting impact of treatment decisions}

Reflection of the cancer treatment decisions highlighted the complexity of managing multiple diseases 'I'm still recovering so I'm more aware of problems...I've come home and having to adapt and things in the house or re-learnt things which I'd forgotten about' (P008M). This may be due to underestimating or lack of communication about the potential severity of treatment side effects, impact on their dementia or quality of life than anticipated during decision-making: 'do I think he had come to terms with [treatment decision]...I don't think he had. I still don't think he has' (H005M:Dr,surgeon). HCPs also reflected on the appropriateness of their clinical decisions and described using these to guide subsequent patient consultations:

...make sure that they're aware from the outset...a very real chance that their dementia will be accelerated by the whole process...my approach to it has been very different (H003F:Dr,consultant)

After cancer treatment, patients and their family needed more information about community-based care. Following surgery, one patient found the number of staff involved in wound care confusing you're swapping about all the time aren't you...you don't seem to have the same one every time' $(\mathrm{P} 007 \mathrm{M})$. One experience of a patient and her son, who decided during the best interest meeting with HCPs not to have cancer treatment, highlighted a gap in the decision-making pathway indicating unmet information needs: 'they didn't really go through what we need to know now... I don't really know what's happening, which isn't a great position to be in' (C010M:son).

\section{DISCUSSION}

This is the first study to explore cancer treatment decision-making in people with cancer-dementia, including HCP perspectives. Results highlight the difficulties experienced by this patient group and underscores the crucial role of informal caregivers in decision-making at all stages. The findings also illustrate the complexities and dilemmas for oncology HCPs in understanding the impact that dementia may have when determining appropriate treatment options, assessing patient understanding and family involvement.

The findings show that patients with cancer-dementia rely greatly on their informal caregivers to support cancer treatment decision-making from the initial cancer diagnosis to follow-up care. Information needs were highlighted at all stages of the cancer pathway echoing research suggesting that people with dementia have difficulty accessing services for non-cancer comorbidities. ${ }^{31}$ The level of functioning including communication ability appeared to influence patient ability for active involvement in cancer treatment decision-making processes; this parallels previous research exploring family member involvement in everyday decision-making in people with dementia and cancer outpatient appointments. ${ }^{22} 32$ Our study adds that patients with dementia require support 
from the beginning of the cancer pathway (reaching a diagnosis) and following treatment. In particular, it highlights the importance of family involvement in supporting people with cancer-dementia also known both in other aspects of dementia care and cancer treatment decision-making when there are no comorbid conditions. ${ }^{15} 33$

Although attempts were made to involve this patient sample in treatment decision-making, it appeared that HCPs had difficulties ensuring the patient had an 'active' role in consultations due to communication difficulties. This draws parallels with perspectives of palliative care HCPs in the context of dementia and elderly cancer patient populations. ${ }^{34}{ }^{35}$ Linked to this, HCPs highlighted that involvement of healthcare staff with dementia expertise was reassuring by providing advice on decision-making and supporting the patient through treatment. However, information about the functional impact of dementia, and thus the impact on cancer treatment understanding and compliance, was often not known until the patient and HCP met to discuss treatment options. This suggests that the individual impact of dementia should ideally be known at initial MDT meetings. Such factors were recommended as useful at this stage from an ethnographic study of oncology MDTs focused on complex, older patients. ${ }^{36}$ HCPs spoke about their experience of cancer-dementia in general terms although this may reflect the relatively short period of time oncology professionals spend with patients during treatment decision-making. Prospective longer term studies would facilitate gathering these perspectives and how they may change over time.

Similarly, HCPs suggested that dementia-related cognitive and communication impairments influence treatment options in relation to potential side effects and appropriate management. It is known that it is more difficult to identify and manage pain for patients with cancer-dementia ${ }^{37}$ and so these findings illustrate the complex nature of decision-making in this context. Additionally, dementia has been recognised by oncology HCPs as adding an extra layer of patient vulnerability when caring for cancer patients. ${ }^{39} 40$ The need for hospital admissions, including those that are unplanned, can have a negative impact on a patient's dementia, which may lead to declined cognitive and functional ability, and quality-of-life deterioration. ${ }^{41}$ Consequently, it is crucial that this is considered during decision-making processes. These findings are likely to transfer to other health conditions that coexist with dementia and it is vital to consider the length of consultations between healthcare professionals and people with dementia regardless of the context.

These findings highlight that patients and their family can have ongoing information needs following the completion of treatment, particularly when the patient's dementia has progressed. Given that people with dementia experience delays in cancer diagnosis with late-stage presentation limiting treatment options, ${ }^{21}$ this raises questions regarding identification and assessment in primary care, referrals and decision-making processes across primary-secondary, and secondary-tertiary care. Recent multimorbidity guidelines emphasise the importance of shared decision-making and opportunities for patients to discuss values, priorities and goals. ${ }^{42}$ However, people with cancer-dementia may lack capacity to do this and rely on family members to act on their behalf. Future research should focus on the cancer pathway at initial symptom recognition and primary care referral in order to explore the decisions made at this stage. Further work is required to develop specific support for this growing patient group including informal caregivers.

\section{Strengths and limitations}

This study provides a rich and varied reflection of the accounts of patients with cancer-dementia, their informal caregivers and oncology HCPs who underwent cancer treatment decision-making. Although the analysis relied predominantly on informal caregiver perspectives, attempts were made to involve patients with dementia, irrespective of dementia severity. However, patients' memories of cancer treatment, including decision-making and their ability to verbalise it, were limited.

While this study captured the views of HCPs from one cancer centre, this was post cancer diagnosis and primary or secondary care HCP perspectives were not captured. However, our findings revealed that tertiary care clinicians would value collaboration with primary/secondary care regarding the provision of care for patients with cancer-dementia. Similarly, although we asked participants about their experiences of decision-making within primary-secondary care, it was not possible to recruit patients and caregivers before they attended the cancer centre. Given the heterogeneity of the patient sample in relation to both dementia and cancer diagnosis, it was not possible to identify particular discrepancies across the sample. However, this study highlights the complexity of supporting this particular patient group.

\section{Conclusions}

While cancer and comorbidities are complex scenarios, dementia presents particular clinical challenges in relation to patient understanding and decision-making. It is clear that this patient group and their family require specific support when faced with cancer treatment decisions. Insight into oncology HCP perspectives provided evidence of dementia-specific considerations for cancer treatment options suggesting that appropriate models of care are required. Future research is essential to develop suitable interventions for this complex patient group before, during and after cancer treatment.

Acknowledgements The authors would like to sincerely thank the staff, patients and family involved in this research.

Contributors Conception and design: LM, CF, JK, LB, CS, GG and JY; data collection: LM, CF and LB; data analysis and interpretation: LM, CF, JK, LB, CS, GG, JY and SB; drafting of the paper: LM, CF, JK, CS, GG, LB, SB and JY; all authors reviewed the manuscript and approved the final draft.

Funding This work was supported by Manchester Institute for Collaborative Research on Ageing. 


\section{Competing interests None declared.}

Patient consent Detail has been removed from this case description/these case descriptions to ensure anonymity. The editors and reviewers have seen the detailed information available and are satisfied that the information backs up the case the authors are making.

Ethics approval Yorkshire \& The Humber—Bradford Leeds Research Ethics Committee (ref: 16/YH/0156).

Provenance and peer review Not commissioned; externally peer reviewed.

Data sharing statement Additional unpublished data from the study are not available.

Open Access This is an Open Access article distributed in accordance with the Creative Commons Attribution Non Commercial (CC BY-NC 4.0) license, which permits others to distribute, remix, adapt, build upon this work non-commercially, and license their derivative works on different terms, provided the original work is properly cited and the use is non-commercial. See: http://creativecommons.org/ licenses/by-nc/4.0/

(c) Article author(s) (or their employer(s) unless otherwise stated in the text of the article) 2018. All rights reserved. No commercial use is permitted unless otherwise expressly granted.

\section{REFERENCES}

1. Collerton J, Davies K, Jagger C, et al. Health and disease in 85 year olds: baseline findings from the Newcastle $85+$ cohort study. BMJ 2009;339:b4904.

2. Alzheimer's Society. Dementia UK: Update. London, 2014.

3. Cancer Research UK. Cancer incidence by age. Secondary Cancer incidence by age. 2015. http://www.cancerresearchuk.org/healthprofessional/cancer-statistics/incidence/age\#heading-Zero\# VUVKIf7RwvZVzEZR.97

4. Maddams J, Utley M, Møller H. Projections of cancer prevalence in the United Kingdom, 2010-2040. Br J Cancer 2012;107:1195-202.

5. Balducci L, Colloca G, Cesari M, et al. Assessment and treatment of elderly patients with cancer. Surg Oncol 2010;19:117-23.

6. Kedia SK, Chavan PP, Boop SE, et al. Health care utilization among elderly medicare beneficiaries with coexisting dementia and cancer. Gerontology and Geriatric Medicine 2017;3:233372141668904.

7. Wongrakpanich S, Hurst A, Bustamante J, et al. Prognostic significance of dementia in older adults with solid tumors. Dement Geriatr Cogn Disord 2017;43:38-44.

8. Hopkinson JB, Milton R, King A, et al. People with dementia: what is known about their experience of cancer treatment and cancer treatment outcomes? A systematic review. Psychooncology 2016;25:1137-46.

9. Kehl KL, Landrum MB, Arora NK, et al. Association of actual and preferred decision roles with patient-reported quality of care: shared decision making in cancer care. JAMA Oncol 2015;1:50-8.

10. Kane HL, Halpern MT, Squiers LB, et al. Implementing and evaluating shared decision making in oncology practice. CA Cancer J Clin 2014;64:377-88.

11. Taylor C, Finnegan-John J, Green JS. "No decision about me without me" in the context of cancer multidisciplinary team meetings: a qualitative interview study. BMC Health Serv Res 2014;14:488.

12. Stacey D, Paquet L, Samant R. Exploring cancer treatment decisionmaking by patients: a descriptive study. Curr Oncol 2010;17:85-93.

13. Smebye KL, Kirkevold M, Engedal K. How do persons with dementia participate in decision making related to health and daily care? A multi-case study. BMC Health Serv Res 2012;12:241.

14. Taghizadeh Larsson A, Österholm JH. How are decisions on care services for people with dementia made and experienced? A systematic review and qualitative synthesis of recent empirical findings. Int Psychogeriatr 2014;26:1849-62.

15. Miller LM, Whitlatch CJ, Lyons KS. Shared decision-making in dementia: a review of patient and family carer involvement. Dementia 2016;15:1141-57.

16. Hobbs GS, Landrum MB, Arora NK, et al. The role of families in decisions regarding cancer treatments. Cancer 2015;121:1079-87.
17. DAVIES SUE, Nolan M. 'Making the best of things': relatives' experiences of decisions about care-home entry. Ageing Soc 2003;23:429-50.

18. Lord K, Livingston G, Cooper C. A systematic review of barriers and facilitators to and interventions for proxy decision-making by family carers of people with dementia. Int Psychogeriatr 2015;27:1301-12.

19. Elliott BA, Gessert CE, Peden-McAlpine C. Family decision-making in advanced dementia: narrative and ethics. Scand J Caring Sci 2009;23:251-8.

20. Caron CD, Griffith J, Arcand M. End-of-life decision making in dementia: the perspective of family caregivers. Dementia 2005;4:113-36.

21. McWilliams L, Farrell C, Grande G, et al. A systematic review of the prevalence of comorbid cancer and dementia and its implications for cancer-related care. Aging Ment Health 2017:1-18.

22. Courtier N, Milton R, King A, et al. Cancer and dementia: an exploratory study of the experience of cancer treatment in people with dementia. Psychooncology 2016;25:1079-84.

23. Wong RE, Weiland TJ, Jelinek GA. Emergency clinicians' attitudes and decisions in patient scenarios involving advance directives. Emerg Med J 2012;29.

24. Hamaker ME, Hamelinck VC, van Munster BC, et al. Nonreferral of nursing home patients with suspected breast cancer. J Am Med Dir Assoc 2012;13:464-9.

25. Woods B, Pratt R. Awareness in dementia: ethical and legal issues in relation to people with dementia. Aging Ment Health 2005;9:423-9.

26. Murphy K, Jordan F, Hunter A, et al. Articulating the strategies for maximising the inclusion of people with dementia in qualitative research studies. Dementia 2015;14:800-24.

27. Slaughter S, Cole D, Jennings E, et al. Consent and assent to participate in research from people with dementia. Nurs Ethics 2007; 14:27-40.

28. British Psychological Society. Conducting research with people not having the capacity to consent to their participation: A practical guide for researchers. Leicester: British Psychological Society, 2008.

29. Braun V, Clarke V. Using thematic analysis in psychology. Qual Res Psychol 2006;3:77-101.

30. Gale NK, Heath G, Cameron E, et al. Using the framework method for the analysis of qualitative data in multi-disciplinary health research. BMC Med Res Methodol 2013;13:117.

31. Bunn F, Burn AM, Robinson L, et al. Healthcare organisation and delivery for people with dementia and comorbidity: a qualitative study exploring the views of patients, carers and professionals. BMJ Open 2017;7:e013067

32. Samsi K, Manthorpe J. Everyday decision-making in dementia: findings from a longitudinal interview study of people with dementia and family carers. Int Psychogeriatr 2013;25:949-61.

33. Shin DW, Cho J, Roter DL, et al. Preferences for and experiences of family involvement in cancer treatment decision-making: patientcaregiver dyads study. Psychooncology 2013;22:2624-31.

34. Mcllfatrick S. Assessing palliative care needs: views of patients, informal carers and healthcare professionals. J Adv Nurs 2007;57:77-86.

35. Amalraj S, Starkweather C, Nguyen C, et al. Health literacy, communication, and treatment decision-making in older cancer patients. Oncology 2009;23:369-75.

36. Bridges J, Hughes J, Farrington N, et al. Cancer treatment decisionmaking processes for older patients with complex needs: a qualitative study. BMJ Open 2015;5:e009674.

37. Monroe T, Carter M, Feldt K, et al. Assessing advanced cancer pain in older adults with dementia at the end-of-life. J Adv Nurs 2012;68:2070-8.

38. Iritani S, Tohgi M, Miyata $\mathrm{H}$, et al. Impact of dementia on cancer discovery and pain. Psychogeriatrics 2011;11:6-13.

39 Witham G, Haigh C, Foy S. The challenges of health professionals in meeting the needs of vulnerable patients undergoing chemotherapy: a focus group study. J Clin Nurs 2014;23:2844-53.

40. Bartlett A, Clarke B. An exploration of healthcare professionals' beliefs about caring for older people dying from cancer with a coincidental dementia. Dementia 2012;11:559-65.

41. Sampson EL, Blanchard MR, Jones L, et al. Dementia in the acute hospital: prospective cohort study of prevalence and mortality. $\mathrm{Br} \mathrm{J}$ Psychiatry 2009;195:61-6.

42. National Institute for Clinical Excellence. Multimorbidity: Quality Standard [QS153], 2016. 